\title{
Experimental study to evaluate the usefulness of S-1 in a model of peritoneal dissemination of gastric cancer
}

\author{
Takuji Mori, Yoshiyuki Fujiwara, Masahiko Yano, Shigeyuki Tamura, Takushi Yasuda, Shuji Takiguchi, \\ and Morito Monden \\ Department of Surgery and Clinical Oncology, Graduate School of Medicine, Osaka University, 2-2 Yamadaoka (E-2), Suita, \\ Osaka 565-0871, Japan
}

\begin{abstract}
Background. Favorable results have been reported for the novel oral anticancer agent S-1 (TS-1 $\left.{ }^{\circledR}\right)$ in clinical studies of advanced gastric cancer with peritoneal dissemination. In the present study we assessed its pharmacokinetics, inhibitory effects, and effect on survival time in an animal model.

Methods. A model of peritoneal dissemination was created by intraperitoneally implanting 4-week-old female BALBc nu/nu mice with the human gastric cancer cell line MKN-45 after transfection with a fluorescent protein-expressing vector. Pharmacokinetics were investigated by measuring intratumor, peritoneal lining, and blood concentrations after the administration of S-1 and fluorouracil (5-FU). The effect of S-1 on survival time was also assessed, by administration once daily to seven animals per group, starting on day 7 after implantation, and survival time was compared with that of an untreated control group. The inhibitory effect of S-1 on peritoneal dissemination was evaluated by killing mice at the start of administration, and 1 and 3 weeks after the start of administration, and examining them for the presence of peritoneal dissemination under a fluorescence stereomicroscope.

Results. Maintenance of high 5-FU concentrations in the intraperitoneal tumors was confirmed in the $S-1$ group, and survival time was prolonged without any decrease in oral food intake or body weight.

Conclusion. Assessment in a model of peritoneal dissemination of gastric cancer showed that the novel oral anticancer agent $\mathrm{S}-1$ was effective against peritoneal dissemination, and that it improved the survival rate.
\end{abstract}

Key words Gastric cancer · Peritoneal dissemination $\cdot$ S-1

\section{Introduction}

Peritoneal dissemination is the most common form of recurrence after resection of gastric cancer [1,2], and

Offprint requests to: $\mathrm{T}$. Mori

Received: September 25, 2002 / Accepted: February 5, 2003 control of peritoneal recurrence seems to be necessary to improve the prognosis of gastric cancer.

A variety of treatment methods, including extended operations, peritoneal resection, systemic chemotherapy, and intraperitoneal hyperthermic perfusion chemotherapy, have already been tried against peritoneal recurrence [3-8], but none of them has ever been established as a standard treatment.

S-1 is a new dihydropyrimidine dehydrogenase (DPD) inhibitory fluoropyrimidine (DIF) preparation [9]. It improves bioavailability by including gimestat (CDHP), which has DPD-inhibiting action, and otastat potassium (Oxo), which reduces its gastrointestinal toxicity, in the formulation, and makes effective treatment possible. S-1 has recently been reported to have marked efficacy against advanced gastric cancer associated with peritoneal dissemination $[10,11]$, but the basis of the efficacy of S-1 against peritoneal dissemination was unclear.

In this study we conducted an experimental assessment of the effect of S-1 on peritoneal dissemination of gastric cancer in a model of peritoneal dissemination developed in our department [12] (Surgery and Clinical Oncology, Graduate School of Medicine, Osaka University) that enabled observations down to the micrometastasis level, and we investigated its usefulness from a pharmacokinetic standpoint.

\section{Materials and methods}

\section{Cell lines and drugs}

We previously reported a gastric cancer cell line, MKN45-EGFP, which had been transfected with an enhanced green fluorescent protein (EGFP)-expressing plasmid vector [12]. Four-week-old female BALBc nu/nu mice were purchased from Clea Japan (Tokyo, Japan) and maintained under specific-pathogen-free 
conditions. S-1, a pharmaceutical preparation composed of tegafur (FT), gimestat (CDHP, which inhibits 5-FU degradation by the enzyme DPD), and otastat potassium (Oxo, which reduces the gastrointestinal toxicity of 5-FU) was provided by Taiho Pharmaceutical (Tokyo, Japan), and fluorouracil (5-FU) was obtained commercially (Kyowa Pharmaceutical Tokyo, Japan). The maximum tolerated dose (MTD; the highest dose level causing no deaths and a maximum weight loss of $10 \%$ ) of these drugs was determined in preliminary experiments: the MTD of S-1 was $10 \mathrm{mg} / \mathrm{kg}$ body weight and that of 5-FU was $26 \mathrm{mg} / \mathrm{kg}$ body weight $[13,14]$.

\section{Tissue distribution studies}

Measurement of 5-FU and CDHP in tumors, peritoneal lining, and plasma

On day 21 after intraperitoneal (i.p.) implantation of MKN-45-EGFP cells, S-1, at $10 \mathrm{mg} / \mathrm{kg}$ body weight, or $5-\mathrm{FU}$, at $26 \mathrm{mg} / \mathrm{kg}$ body weight, was administered to tumor-bearing mice by gavage. Three mice were allocated to each group for the measurement of 5-FU concentrations. At $0.5,1,2,4$, and $8 \mathrm{~h}$ after $\mathrm{S}-1$ or 5 -FU administration, mice were killed and biological specimens were collected. Blood specimens were collected in centrifuge tubes containing heparin and centrifuged immediately to separate the plasma. Tumor tissues and peritoneal lining were stored at $-80^{\circ} \mathrm{C}$ until use.

5-FU and CDHP concentrations were determined according to the method of Matsushima et al. [15]. After dichloromethane extraction, 5-FU and CDHP were extracted from the residue with ethyl acetate, and the concentrations of 5-FU and CDHP were determined with a negative-ion chemical ionization-gas chromatograph/mass spectrometer, with a stable isotope for each compound as an internal reference. The measurable ranges in the samples were $1-400 \mathrm{ng} / \mathrm{ml}$ plasma and $1-$ $400 \mathrm{ng} / \mathrm{g}$ tumor and peritoneal lining for 5-FU; and 2$800 \mathrm{ng} / \mathrm{ml}$ plasma and $2-800 \mathrm{ng} / \mathrm{g}$ tumor and peritoneal lining for CDHP. Samples containing high concentrations that exceeded the assay range were appropriately diluted before analysis.

\section{Comparison of the therapeutic effects of the oral} anticancer agents $S-1$ and 5-FU

MKN-45-EGFP cells $\left(5 \times 10^{6} /\right.$ mouse) were injected into the peritoneal cavity of 4-week-old female BALBc nu/nu mice on day 0 . Seven mice were allocated to each of two groups, an S-1 group and a 5-FU group, and the drugs were administered by gavage daily at doses of $10 \mathrm{mg} / \mathrm{kg}$ body weight and $26 \mathrm{mg} / \mathrm{kg}$ body weight, respectively, starting on day 7 . The mice were killed on day 21 after tumor cell injection. The antitumor effect of the agents was assessed by counting the number of fluorescent microfoci under a fluorescence stereomicroscope.

\section{Assessment of survival time}

Evaluation of the effect of S-1 on survival time, body weight, and oral intake in the mouse model

Survival time was compared in S-1-treated mice and untreated controls. Body weight and oral intake were also measured at several points to evaluate the toxicity of the agent and the degree of cachexia.

The experimental protocol was approved by the Ethics Review Committee for Animal Experimentation of Osaka University School of Medicine.

\section{Statistical analysis}

Significant differences between the treatment group and untreated controls were assessed using the $t$-test. The log rank test was used to assess significant differences in survival time between groups. $P$ values of less than 0.05 were considered evidence of statistical significance.

\section{Results}

\section{Tissue distribution studies}

\section{5-FU concentration in peritoneal tumors, peritoneal lining, and plasma of mice}

To investigate the pharmacokinetics of S-1, the concentration of 5-FU was determined in tumors, peritoneal lining, and plasma obtained from tumor-bearing mice after the administration of the MTDs of S-1 and 5-FU $[13,14]$. After $8 \mathrm{~h}$, the 5-FU level in the peritoneal lining and plasma of the S-1 group had decreased to $15.7 \pm$ $4.4 \mathrm{ng} / \mathrm{g}$ and $3.0 \pm 2.6 \mathrm{ng} / \mathrm{ml}$, respectively, but it was $427.4 \pm 37.5 \mathrm{ng} / \mathrm{g}$ in the tumors and tended to remain elevated for a longer time in the tumors than in the peritoneal lining and plasma. The area under the curve (AUC) in the tumors was higher than that in the peritoneal lining and plasma.

In comparison with the 5-FU group, the AUC in the S-1 group was 4.2-times higher in the tumors (4421.9 ng/g for S-1 and $1041.8 \mathrm{ng} / \mathrm{g}$ for 5-FU), 5.5-times higher in peritoneal lining $(1365.6 \mathrm{ng} / \mathrm{g}$ for $\mathrm{S}-1$ and $148.5 \mathrm{ng} / \mathrm{g}$ for $5-\mathrm{FU}$ ) and 22 times higher in plasma ( $2510.3 \mathrm{ng} / \mathrm{ml}$ for S-1 and $115.1 \mathrm{ng} / \mathrm{ml}$ for 5-FU) (Fig. 1).

CDHP concentration and distribution in tumors, peritoneal lining, and plasma of mice after administration of $S-1$ or $5-F U$

The mean concentrations of CDHP in plasma and tumors obtained from tumor-bearing mice $(n=3)$ at 0.5 , $1,2,4$, and $8 \mathrm{~h}$ after the administration of $\mathrm{S}-1$ or 5 -FU are shown in Fig. 2. CDHP was poorly distributed in tumors compared with peritoneal lining and plasma at $0.5 \mathrm{~h}$ after administration, but the distribution was approximately the same at $1,2,4$, and $8 \mathrm{~h}$. No CDHP was detected in the tumors or plasma in the 5-FU group (Fig. 2). 

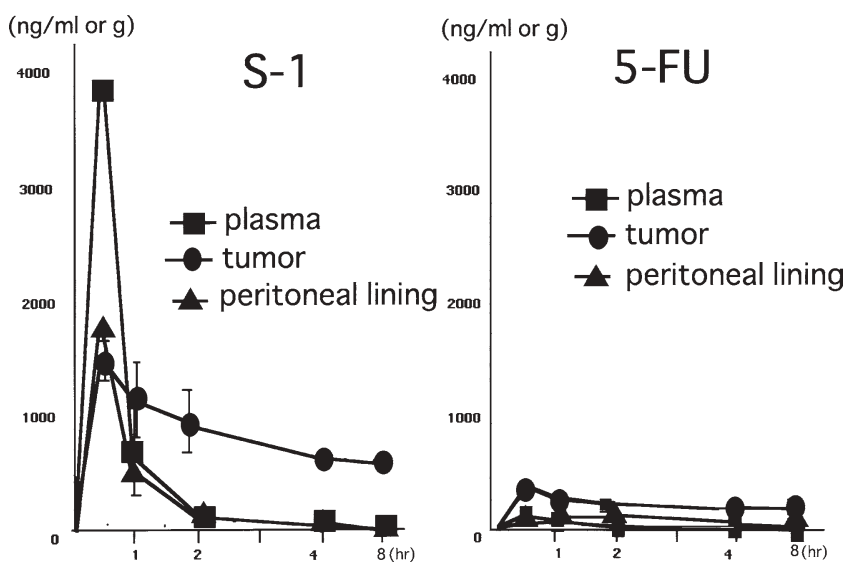

Fig. 1. Levels of 5-fluorouracil $(5-F U)$ in tumor, peritoneal lining, and plasma after the administration of S-1 (TS-1; Taiho Pharmaceutical). S-1 (10 mg/kg) and 5-FU (26 mg/kg) were administered to MKN-45-EGFP-bearing mice. After the administration, tumor, peritoneal lining, and blood samples were obtained at $0.5,1,2,4$, and $8 \mathrm{~h}$. Data values are means \pm $\mathrm{SD}$ of three mice in each group
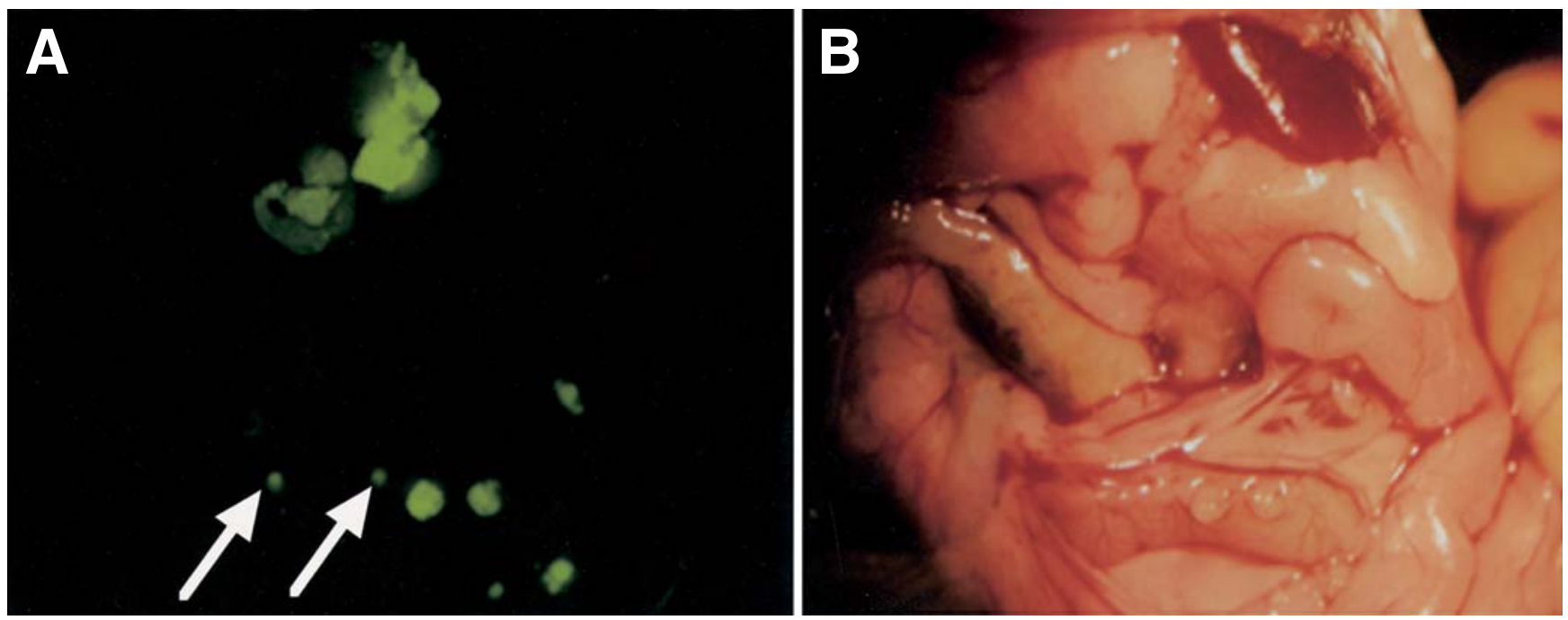

Fig. 3. A Under a fluorescence stereomicroscope, tumors were detected in the greater omentum and mesentery. B No nodules were detected in the same field by macroscopic observation. $\mathbf{A} \times 90$

\section{Comparison of therapeutic effects of the anticancer} agents $S$-1 and 5-FU

Three mice were killed on day 7 after i.p. injection, and peritoneal seeding was confirmed in all three (Fig. 3). On day 21, fluorescence stereomicroscopic examination revealed a significantly lower number of fluorescent foci in the $\mathrm{S}-1$ group $(95.5 \pm 64.2)$ than in the 5 -FU group $(277.0 \pm 177.6 ; P=0.05)$ and the control group $(590.0 \pm$ $118.7 ; P<0.01)$. The number of peritoneal foci in the 5 FU group was significantly lower than that in the control group $(P<0.01$; Fig. 4).

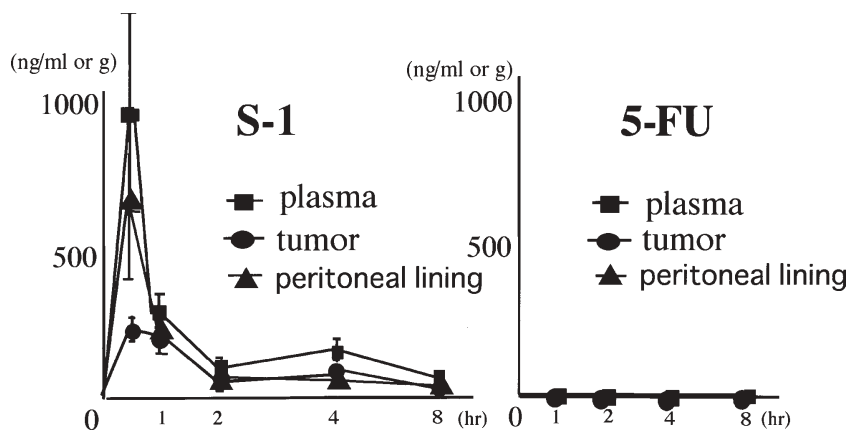

Fig. 2. Levels of gimestat (CDHP) in tumor, peritoneal lining, and plasma after the administration of S-1. S-1 $(10 \mathrm{mg} / \mathrm{kg})$ was administered to MKN-45-EGFP-bearing mice. After administration, tumor, peritoneal lining, and blood samples were obtained at $0.5,1,2,4$, and $8 \mathrm{~h}$. Data values are means \pm $\mathrm{SD}$ of three mice in each group 


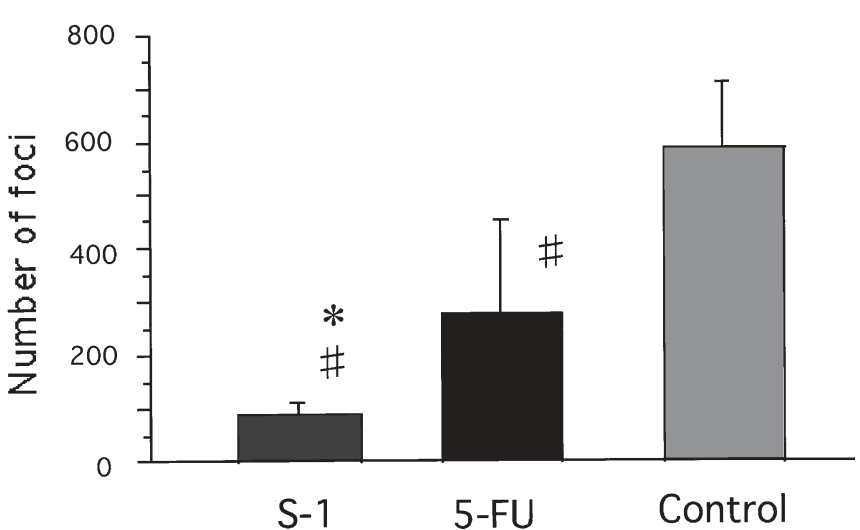

Fig. 4. Comparison of therapeutic effects of oral anticancer agents, S-1 and 5-FU, on MKN-45-EGFP-bearing BALB/c mice. Data values are means $\pm \mathrm{SD}$ of the number of tumors detected by fluorescence stereomicroscope on day 21 in seven mice in each group. ${ }^{\#} P<0.01$ vs control; $* P$ vs 5 -FU

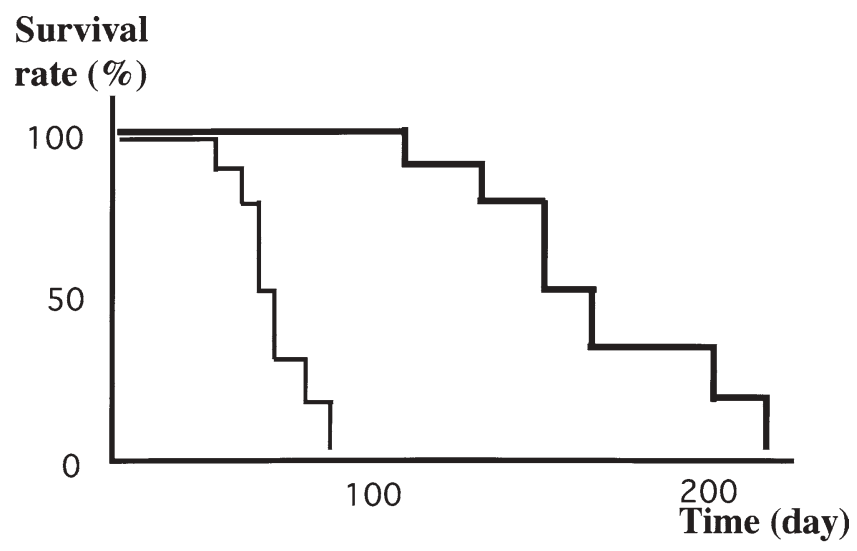

Fig. 5. Survival curves. The survival curves of the control group (thin line) and the S-1-treated group (thick line) were significantly different $(P<0.01 ; n=7$ for each group)

Body weight and food intake of S-1-treated mice Body weight and food intake were greatly diminished from days 56 to 64 in the control group of MKN-45EGFP-bearing mice, but in the S-1-treated group there were no differences in these parameters relative to findings in mice not bearing any tumors (Fig. 6A, B).

\section{Discussion}

The prognosis of patients with peritoneal dissemination of gastric cancer is very poor [1,2], and it was hoped that effective chemotherapy would be established to improve the prognosis. However, although a variety of chemotherapies have been tried, none has ever been established as an effective treatment for peritoneal dissemination [5-8].
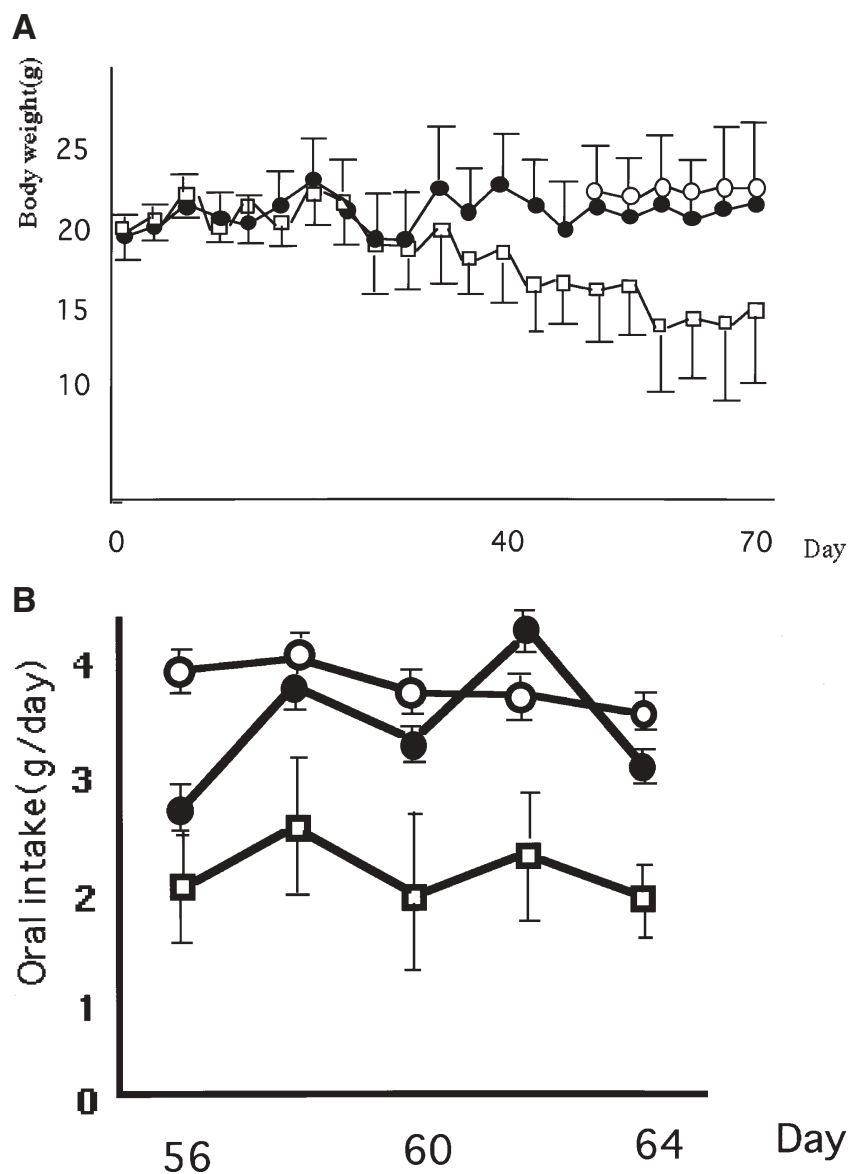

Day

Fig. 6. A. Changes in body weight of mice in the S1-treated and control groups from day 0 to day 70 . B. Comparison of mean oral intake of food between the two groups from day 56 to day 64. Data values are means \pm SD of seven mice in each group. Open circles, Normal (mice not bearing any tumors); closed circles, S-1-treated; open squares, controls

The novel oral anticancer preparation S-1 has recently been reported to be effective against advanced gastric cancer, and there have even been scattered reports of marked efficacy against peritoneal dissemination $[10,11]$.

In this study we conducted a pharmacokinetic investigation to determine whether administration of S-1 would result in the drug reaching tumors resulting from peritoneal dissemination, which are said to be difficult for drugs to reach.

Although the blood concentrations in the S-1 group were high $30 \mathrm{~min}$ after oral administration, they were almost always low at $2 \mathrm{~h}$. These high blood concentrations of 5-FU in the S-1 group might be mainly due to CDHP, which is an effective inhibitor of dihydropyrimidine dehydrogenase (DPD) [9,16,17], strongly inhibiting the degeneration of 5-FU in liver. Moreover, adverse effects were not observed in the S-1 group in spite of the high blood concentration of 5-FU. This might be partly because otastat potassium (Oxо) sup- 
presses gastrointestinal disturbances and other toxic effects caused by the phosphoribosylation of 5-FU in the gastrointestinal tract $[16,18]$. However further examination is necessary to clarify the underlying biochemical mechanism of this action.

In contrast to the blood concentrations, the maximum time (Tmax) of the peritoneal tumor concentrations occurred at $1 \mathrm{~h}$, but high levels were also maintained from $2 \mathrm{~h}$ to $8 \mathrm{~h}$ after administration. This resulted in a high AUC, and the efficacy of S-1 on intraperitoneal tumors is thought to be attributable to the high AUC.

Measurement of the concentration of the DPD inhibitor CDHP at the same times revealed that from $1 \mathrm{~h}$ to $8 \mathrm{~h}$, the concentrations of CDHP in the intraperitoneal tumors were at the same levels as those in other tissues. The fact that CDHP reached the intraperitoneal tumors appears to be related to inhibition of the breakdown of 5-FU and prolonged maintenance of high concentrations of 5-FU in the intraperitoneal tumors.

Next, we examined the suppressive effect on peritoneal dissemination and the effect on survival time. There were significantly fewer intraperitoneal tumor nodules, including those at the level of micrometastases, in the S1 group on day 21 after intraperitoneal implantation, confirming that the inhibition of peritoneal dissemination by S- 1 begins early. Observations of survival time showed that all of the animals in the S-1 group were alive when all animals in the control group had died. At that time there were no changes in oral food intake or body weight in the S-1 group compared with the start of the observation period. When all animals in the control group had died, S-1 administration was stopped, and survival time in the S-1 group was observed. Interestingly, the results showed that the mice in the S-1 group gradually died, with all of them having succumbed by day 60 ; thus, the inhibitory effect on the growth of peritoneal dissemination by S-1 appears to have been partial remission (PR) or a dormant state (no change; NC) and not complete remission (CR). The results of this experiment suggest that, unless adverse effects develop, administration of S-1 as a treatment for peritoneal dissemination should be continued as long as possible.

The absence of any decline in oral food intake during longterm administration of S- 1 is thought to be attributable to the reduction of gastrointestinal tract injury (a common adverse effect of 5-FU) brought about by Oxo. These findings suggest that $\mathrm{S}-1$ is a suitable preparation for longterm oral administration.

To date, chemotherapy has been the predominant modality of treatment for patients with advanced gastric cancer, especially patients with peritoneal dissemination [5-8]. Chemotherapy requires treatment by the intravenous infusion of agents such as 5-FU and cisplatin (CDDP), and hospitalization. It is questionable whether chemotherapy provides satisfactory im- provement of prognosis and quality of life (QOL) in patients with advanced gastric cancer, whose general condition is poor and whose remaining time is limited. Even if intensive inpatient chemotherapy is carried out and PR is obtained, the cancer rapidly progresses as soon as the patient is discharged, because it is impossible to continue such treatment in the outpatient clinic. Although the efficacy rate $(\mathrm{PR}+\mathrm{CR})$ is high, there is no prolongation of survival time in the end.

In contrast, because S-1 can be administered orally, treatment on an outpatient basis is possible, without any need for hospitalization, and because the clinic visits are also short, high patient QOL can be maintained. Moreover, from the standpoint of economics, which has become an issue recently, this treatment is beneficial because of the low cost.

Because of 5-FU-degrading enzymes, etc., blood concentrations as stable as those achieved by intravenous infusion generally cannot be obtained with oral medication, and because absorption from the gastrointestinal tract is not steady, diarrhea develops due to gastrointestinal toxicity, and discontinuation of treatment becomes inevitable. However, the S-1 formulation contains a DPD inhibitor, gimestat (CDHP), and an inhibitor of the 5-FU phosphorylating enzyme pyrimidine phosphoribosyltransferase, otostat potassium (Oxo), in a 1:0 and 4:1 ratio with FT; S-1 was developed as an oral preparation designed to achieve high blood 5-FU concentrations and reduced gastrointestinal toxicity. The efficacy of S-1 on various malignant tumors has been shown in clinical studies. The efficacy rate in a late phase II study of stomach cancer was especially favorable, at $49 \%$, and because the incidence of grade 3 or higher adverse events was about $20 \%$ [19], it was enthusiastically prescribed after its marketing in 1999. In the future it may become a first-line drug for advanced gastric cancer.

It seems that, in the future, by assessing combinations of S-1 and other drugs, or by establishing drugsensitivity testing methods, it will become possible to construct a treatment strategy for peritoneal dissemination, for which there is no established method of treatment to date.

In conclusion, we confirmed the pharmacokinetics and growth-inhibiting effect of the oral anticancer agent S-1 on peritoneal dissemination in a model of peritoneal dissemination of gastric cancer, and we determined that S-1 administration prolonged survival time. These findings suggest that S-1 may be effective therapy for the peritoneal dissemination of gastric cancer.

\section{References}

1. Dupont JB, Lee JR, Burton GR, Cohn I Jr. Adenocarcinoma of the stomach: review of 1497 cases. Cancer 1978;41:941-7. 
2. Yamada E, Miyaishi S, Nakazato H, Kato K, Kito T, Takagi H, et al. The surgical treatment of cancer of the stomach. Int Surg 1980;65:387-99.

3. Fujimura T, Yonemura Y, Nakagawara H, Kitagawa H, Fushida S, Nishimura G, et al. Subtotal peritonectomy with chemohyperthermic peritoneal perfusion for peritonitis carcinomatosa in gastrointestinal cancer. Oncol Rep 2000;7:809-14.

4. Yonemura Y, Fujimura T, Fushida S, Fujita H, Bando E, Nishimura G, et al. A new surgical approach (peritonectomy) for the treatment of peritoneal dissemination. Hepatogastroenterology 1999;46:601-9.

5. Yonemura Y, Fujimura T, Fushida S, Takegawa S, Kamata T, Katayama K, et al. Hyperthermo-chemotherapy combined with cytoreductive surgery for the treatment of gastric cancer with peritoneal dissemination. World J Surg 1991;15:530-6.

6. Yu W, Whang I, Suh I, Averbach A, Chang D, Sugarbaker PH. Prospective randomized trial of early postoperative intraperitoneal chemotherapy as an adjuvant to resectable gastric cancer. Ann Surg 1998;228:347-54.

7. Fujimura T, Yonemura Y, Fushida S, Urade M, Takegawa S, Kamata $\mathrm{T}$, et al. Continuous hyperthermic peritoneal perfusion for the treatment of peritoneal dissemination in gastric cancers and subsequent second-look operation. Cancer 1990;65:6571.

8. Shimada K, Ajani JA. Adjuvant therapy for gastric carcinoma patients in the past 15 years: a review of western and oriental trials. Cancer 1999;86:1657-68.

9. Shirasaka T, Shimamoto Y, Ohshimo H, Yamaguchi M, Kato T, Yonekura K, Fukushima M. Development of a novel form of an oral 5-fluorouracil derivative (S-1) directed to the potential of the tumor selective cytotoxicity of 5-fluorouracil by two biochemical modulators. Anticancer Drugs 1996;7:548-57.

10. Iwazawa T, Kinuta M, Yano H, Matsui S, Tamagaki S, Yasue A, et al. An oral anticancer drug, TS-1, enabled a patient with advanced gastric cancer with Virchow's metastasis to receive curative resection. Gastric Cancer 2002;5:96-101.
11. Iwahashi M, Nakamori M, Tani M, Yamaue H, Sakaguchi S, Nakamura M, et al. Complete response of highly advanced gastric cancer with peritoneal dissemination after new combined chemotherapy of S-1 and low-dose cisplatin: report of a case. Oncology 2001;61:16-22.

12. Kaneko K, Yano M, Monden M. Establishment of a visible peritoneal metastatic model from a gastric adenocarcinoma cell line by green fluorescent protein. Int J Oncol 2000;16:893-8.

13. Fukushima M. Preclinical antitumor efficacy of S-1: a new oral formulation of 5-fluorouracil, on human tumor xenografts. Int J Oncol 1998;13:693-8.

14. Unemi N, Takeda S, Tajima K, Kawaguchi Y, Yasuda A. Effect of coadministration of uracil on the antitumor activity of 1(Terahydro-2-furanyl)-5-fluorouracil and the level of 5-fluorouracil in AH 130 bearing Rats. Chemotherapy 1981;29:164-75.

15. Matsushima E, Yoshida K, Kitamura R, Yoshida K. Determination of S-1 (combined drug of tegafur, 5-chloro-2,4dihydroxypyridine and potassium oxonate) and 5-fluorouracil in human plasma and urine using high-performance liquid chromatography and gas chromatography-negative ion chemical ionization mass spectrometry. J Chromatogr B 1997;691:95-104.

16. Takechi T, Nakano K, Uchida J, Mita A, Toko K, Takeda S, et al. Antitumor activity and low intestinal toxicity of S-1, a new formulation of oral tegafur, in experimental tumor models in rats. Cancer Chemother Pharmacol 1997;39:205-11.

17. Tatsumi K, Fukushima M, Shirasaka T, Fujii S. Inhibitory effects of pyrimidine, barbituric acid and pyridine derivatives on 5fluorouracil degradation in rat liver extracts. Jpn J Cancer Res 1987;78:748-55.

18. Shirasaka T, Shimamoto Y, Fukushima M. Inhibition by oxonic acid of gastrointestinal toxicity of 5-fluorouracil without loss of its antitumor activity in rats. Cancer Res 1993;53:4004-9.

19. Koizumi W, Kurihara M, Nakano S, Hasegawa K. Phase II study of S-1, a novel oral derivative of 5-fluorouracil, in advanced gastric cancer. The S-1 Cooperative Gastric Cancer Study Group. Oncology 2000;58:191-7. 